\title{
In vitro analysis of various cell lines responses to electroporative electric pulses by means of electrical impedance spectroscopy
}

Tomás García-Sánchez ${ }^{1}$, Ramon Bragós ${ }^{2}$, Lluis M. Mir ${ }^{1}$

${ }^{1}$ Vectorology and Anticancer Therapies, UMR 8203, CNRS, Univ. Paris-Sud, Gustave Roussy, Université Paris-Saclay, 94805 Villejuif, France.

${ }^{2}$ Electronic and Biomedical Instrumentation Group, Department of Electronic

Engineering, Universitat Politècnica de Catalunya, Barcelona, Spain 


\section{Introduction}

The use of high intensity electric fields for the permeabilization of biological membranes known as electroporation represents nowadays a widespread technique used in very different applications (Andrei $\mathrm{G}$ Pakhomov and Marko S Markov and Andrei G Pakhomov and Marko S Markov, 2010; Yarmush et al., 2014). From its in vitro applications in routine laboratory protocols, mainly as a physical gene transfection method (Andre and Mir, 2004), to its clinical use in the treatment of cancer (Marty et al., 2006; Mir et al., 1991), electroporation has reached in the last ten years a remarkable state of development. However, some questions about the fundamental aspects of the phenomenon are still unanswered. In this direction, the development of reliable methods for monitoring the success of the electroporation treatment both in vitro and in vivo is still necessary. The goal of these methods should be enabling the possibility of studying in a precise way the effects of electric field pulse parameters, or other experimental conditions, and the determination of the extent of membrane permeabilization in a precise way.

The most extended methods for the detection and quantification of electroporation are based in the study of the transport of different molecular species across the electropermeabilized membrane (Batista Napotnik and Miklavčič, 2018; Pucihar et al., 2008), specially, the incorporation of non-permeant exogenous molecules such as fluorescent dyes, ions, cytotoxic agents or DNA plasmids, among others. Coupled with these molecules, a suitable detection method must be used. These methods are mostly based on chemical and physical phenomena, mainly optical, and can detect with high precision very low levels of the transported molecules, and thus of membrane electropermeabilization (Kennedy et al., 2008). However, the drawback of these techniques, considered as non-label free methods, is the need of a reporter molecule that can interfere with the normal development and subsequent use of the treated cells.

Alternatively, label free methods have been developed to measure electropermeabilization. In this case no additional molecule is needed to detect the formation of membrane pores, thus, cells can be normally used after electric field exposure. Among these methods, the leaking of endogenous molecules has been used to study cell permeabilization (Zhan et al., 2010). Also, and although it is not possible to obtain a quantitative information about the extent of permeabilization, the detection of cell swelling after pulse application has been also used as an indicator of successful electroporation (Romeo et al., 2013).

In this direction, an important family of label-free methods based in the measurement of the electrical properties of the sample under treatment have been extensively used (Castellví et al., 2017). An abrupt change in the electrical conductivity of the cell membrane in response to pulse application has been 
observed in individual cells by patch clamp methods (O’Neill and Tung, 1991; Wegner et al., 2013), or by measuring changes in the applied current-voltage waveforms, in cell suspensions (Kinosita Jr and Tsong, 1979; Pavlin et al., 2007) or tissues (Davalos et al., 2004; Ivorra and Rubinsky, 2007). The mentioned change in conductivity involves also a change in the complex electrical impedance of the biological samples that has been also studied at single or multiple frequencies (García-Sánchez et al., 2015; Ivorra et al., 2009; Pasqualotto et al., 2012; Pliquett et al., 1995; Stolwijk et al., 2011).

Traditional multifrequency measurements, also known as electrical impedance spectroscopy (EIS), are based on sweeping the measurement signal frequency over a range between the $\mathrm{kHz}$ and $\mathrm{MHz}$ covering the well-known $\beta$ dispersion of biological tissues (Foster and Schwan, 1989; Martinsen, 2000; Schwan, 1994). However, this approach is time consuming and not valid for monitoring the fast processes that occur during electroporation. Other time-domain methods using broadband signals have been previously proposed to study the fast dynamical phenomena during electroporation (García-Sánchez et al., 2015; Garner et al., 2004; Pliquett and Schoenbach, 2009; Yuxiang et al., 2011). Thanks to the possibility of acquiring immediate information from the system, these methods represent an optimal option specially for real time applications. Some previous studies have already proposed to use a real-time control based on electrical measurements. This was applied to non-viral gene therapy used as a predictor of the extent of permeabilization and as a real-time optimization of the delivered electric field (Cukjati et al., 2007). Other authors also applied a feedback control system to deliver the appropriate electric field to single cells based on electrical measurements (Khine et al., 2007).

In this work, the use of a fast multisine-based approach previously published (García-Sánchez et al., 2015) is extended to three different cell lines. The fast EIS measurements ( 1 spectrum per millisecond) are performed during the interpulse electroporation interval, with the aim of studying the dynamics of the resealing processes. The comparison of the impedance response of the different cell lines brings important information about the general performance of the system and about the sensitivity of impedance in different scenarios. First, the magnitude and phase angle of impedance are shown at different individual frequencies. Subsequently, in order to take advantage of the complete spectral information, data modeling is applied to measurements. The goal of this study is also to give some insights in the information that full spectral information can provide in comparison with single frequency measurements. 


\section{Materials and Methods}

\subsection{Cells and chemicals}

C2C12 mouse myoblasts and HeLa human cervix carcinoma cells were seeded into 24 muti-well plates at an initial density of $40 \times 10^{3}$ cells/well and $50 \times 10^{3}$, respectively, in Dulbecco's Modified Eagle's Medium (DMEM; High Glucose, GlutaMAX Supplement, pyruvate. Gibco, Carlsbad, CA, USA) supplemented with $10 \%$ fetal bovine serum (FBS) and $1 \%$ penicillin-streptomycin.

In the case of DC-3F chinese hamster fibroblasts, cells were seeded at an initial density of $350 \times 10^{3}$ cells/well in Minimal Esential Medium (MEM; GlutaMAX. Gibco, Carlsbad, CA, USA) supplemented with $10 \%$ fetal bovine serum (FBS) and 1\% penicillin-streptomycin. All cells were kept in an incubator at $37{ }^{\circ} \mathrm{C}$ under a $5 \% \mathrm{CO} 2$ humidified atmosphere for $24 \mathrm{~h}$ until full confluence.

A low-conductivity electroporation (LCE) buffer consisting of $250 \mathrm{mM}$ sucrose, $10 \mathrm{mM}$ Tris and $1 \mathrm{mM}$ $\mathrm{MgCl} 2(\mathrm{pH} \mathrm{7}$, osmolarity $287 \mathrm{mmol} / \mathrm{kg}$, conductivity $0,1 \mathrm{~S} / \mathrm{m}$ ) was used during the electroporation pulse delivery and impedance measurements.

Finally, as a permeabilization reporter, fluorescent nucleic acids stain Yo-Pro-1 Iodide ( $\lambda e x=491 \mathrm{~nm}$, $\lambda e m=509 \mathrm{~nm}$, Life technologies, Saint Aubin, France) was added to the permeabilization buffer at a final concentration of $1 \mu \mathrm{M}$.

\subsection{Experimental setup}

\subsubsection{Microelectrodes}

A custom-built microelectrode assembly was used to perform both EIS measurements and electroporation of the adherent cell monolayers. Full details of the microelectrode assembly geometry, its fabrication procedure and its performance can be found in (T. García-Sánchez et al., 2014). The design was conceived to fit in standard 24 multi-well plates. Shortly, the microelectrode structure consisted in six equally-spaced planar electrodes coiled in parallel to form six parallel spirals able to apply electroporation pulses and to perform four-electrode EIS measurements in the cells monolayer. The dimensions of the lines were $75 \mu \mathrm{m}$ in width and there was a $150 \mu \mathrm{m}$ spacing between them. They were fabricated using a low-cost modified 
printed circuit board (PCB) procedure. The microelectrodes were designed to be momentarily positioned above the cell monolayer during the electroporation/EIS measurements procedure at a short distance (10 $\mu \mathrm{m})$ above the cell monolayer during the electroporation/EIS measurements. This was achieved by means of punctual microseparators patterned in the electrodes surface which ensured no direct contact between the cells and the electrodes. A schematic representation of the concept is depicted in Fig. 1a.

\subsubsection{Impedance measurements}

The hardware used to perform the measurements was similar to the one described in full detail in (GarcíaSánchez et al., 2015). Briefly, we used a custom measuring setup based on a PXI platform (National Instruments) comprising an arbitrary waveform generator, a two channels digitizer and a high density multiconfiguration multiplexer matrix. Additionally, a custom made analog front-end was used to interface the measuring signals with the electrodes using a four electrode topology. Commutation between electroporation and measurement connections was synchronously performed with a hardware-based trigger signal provided by the custom-built pulse generator employed. The generator delivered bipolar pulses, 100 $\mu$ s positive $+100 \mu$ s negative in duration, of different amplitudes at a frequency of $1 \mathrm{~Hz}$.

Given the need for a fast impedance measuring approach, we used a time-domain technique based on the use of a broadband multisine signal as excitation. Specifically, the multisine burst consisted of 21 frequencies following a Bilateral Quasi-Logarithmic (BQL) distribution from $5 \mathrm{kHz}$ to $1.313 \mathrm{MHz}$ and had

a duration of $1 \mathrm{~ms}$. All the electroporation/measurements sequences began with 100 continuous initial preelectroporation measurements (total duration of $100 \mathrm{~ms}$ ). Subsequently, the pulse generation was initiated and the measurement system disconnected. When the trigger signal from the pulse generator was detected by the PXI-2530 system, the connections were switched to the measuring system after a fixed delay of 15 $\mathrm{ms}$, and 860 multisine bursts were continuously generated and acquired. The connections were again switched to the pulse generator and the system waited for the next pulse (125 ms). Each sequence was repeated until completing 8 pulses. A custom software was developed in LabView (National Instruments) to control the whole system. The sequence of electroporation and measurement signals is detailed in Fig. $1 b$.

Compensation for the effects of the system frequency response produced by the microelectrodes, cables, amplifiers, etc., was achieved by means of a three-references calibration method [39]. The references were obtained by measuring, with the same setup, saline solutions with three different conductivities in the range of $0.1 \mathrm{~S} / \mathrm{m} \pm 70 \%$. 


\subsection{Experimental procedure}

Previous to the experiment, the 24 multi-well plate was removed from the incubator and the cells washed once with LCE buffer. Subsequently, $200 \mu \mathrm{LCE}+$ Yo-Pro-1 (final concentration $1 \mu \mathrm{M}$ ) were added. Cells were left during $10 \mathrm{~min}$ for stabilization. After this period, the microelectrode assembly was positioned manually above the cell monolayer by means of a custom built applicator. Immediately after, the electroporation-measurements sequence was initiated as described above. The experiments were performed in a controlled temperature chamber at $37^{\circ} \mathrm{C}$. Once the sequence was finished, the microelectrodes were carefully removed and cells were left during 10 additional minutes for resealing. Afterwards, cells were washed twice with PBS and replaced with fresh culture medium before inspection under inverted microscope Zeiss Axio Observer Z1 (Carl Zeiss, Jena, Germany) to detect Yo-Pro-1 fluorescence.

In order to quantify the extent of permeabilization, at least three different fields of each sample were recorded under fluorescent microscope and subsequently analyzed with the image processing free software ImageJ (MacBiophotonics). In order to have an statistical measure of the significance of differences, unpaired Student's t-tests (significance level $5 \%$ ) were performed. 
a)
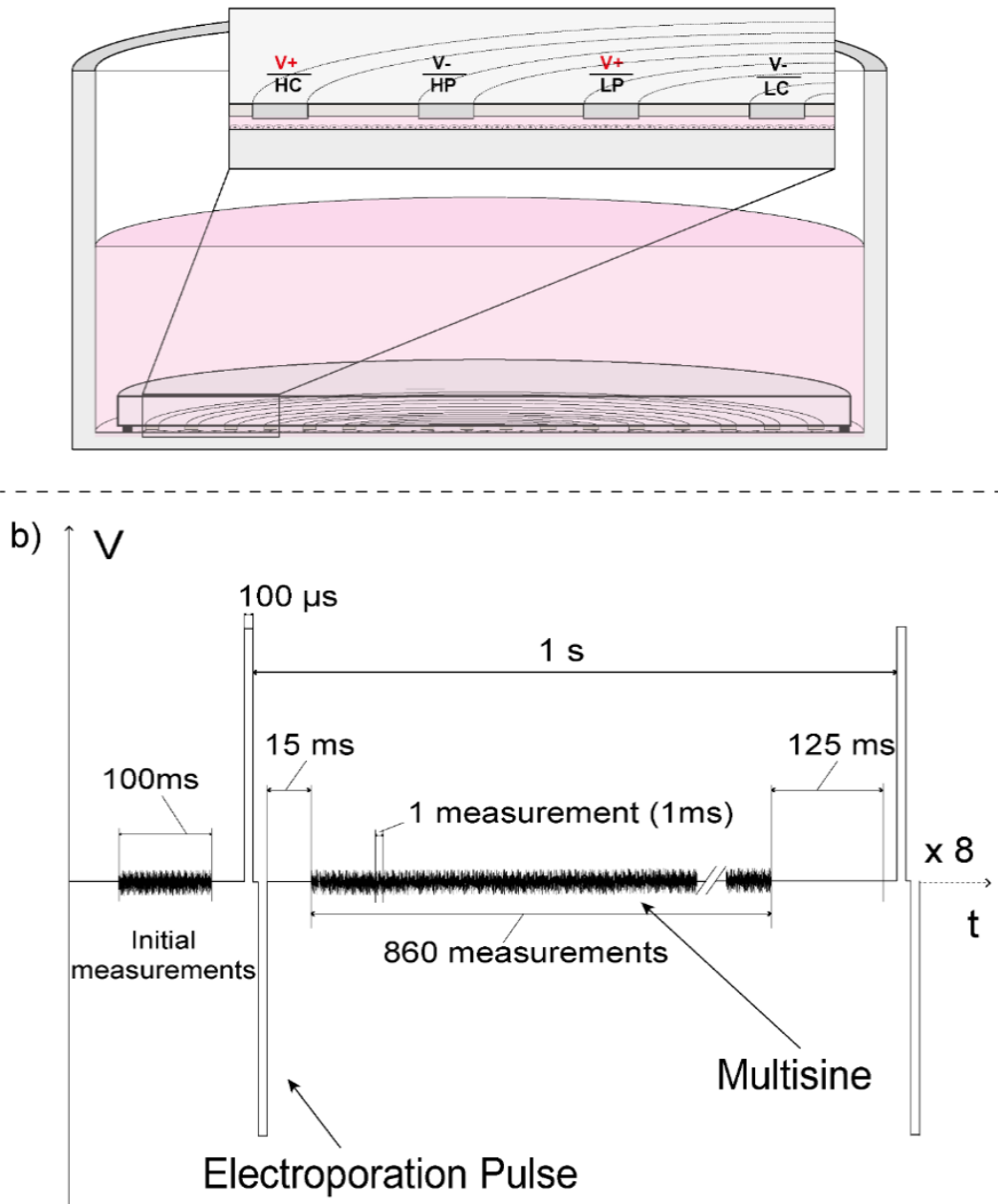

Fig.1. Experimental setup. a) Schematic drawing of the spiral electrode assembly used both for the electroporative electric pulses application and the impedance measurements as detailed in (Tomás GarcíaSánchez et al., 2014). b) Measurement/electroporation sequence used in the experiments (time and voltage are not to scale). 


\section{Results and Discussion}

\subsection{Pre-electroporation measurements}

In this section, the measurements performed in each cell line prior to electroporation pulse application (100 ms initial measurements) are shown. Fig. 2a shows an example of the impedance spectrum displayed by each of the three cell lines under analysis. The Bode plots of the magnitude and phase of the impedance show the typical response of a living tissue and it can be noticed how each cell line has a differential impedance signature demonstrating how the system is capable of detecting the cell monolayers and differences between monolayers from different cell types.

In Fig. $2 b$ a representative micrograph of each cell line is shown. It can be observed at a glance that there exist significant differences in dimensions and organization of the cells. While DC-3F cells are small, with an almost round geometry, and very homogeneously distributed, C2C12 and HeLa cells are much more elongated with clearly a long and a short axis. In the special case of the $\mathrm{C} 2 \mathrm{C} 12$ cells it can also be observed that there are small regions where cells are oriented in a preferential direction.

These initial multifrequency measurements were fitted to the well-known Cole model. In the frequency band ranging from some $\mathrm{kHz}$ to some $\mathrm{MHz}$ corresponding to the $\beta$ dispersion, biological tissues usually display one single relaxation following Cole equation (eq. 1). From the parameters of this mathematical model $\left(R_{0}, R_{\infty}, \alpha\right.$ and $\left.f_{c}\right)$, it has been shown that $\alpha$ and the characteristic frequency $\left(f_{c}\right)$ are directly related with the intrinsic properties of the biological sample under study [8-11]. Specifically, it has been suggested that the characteristic frequency (the frequency corresponding to the maximum in the imaginary part of the impedance) is intimately related to cell size and shape [10]. An example of the fitted model and the extracted $\mathrm{f}_{\mathrm{c}}$ is shown in Fig. 2c (upper insert).

$$
Z_{\text {cole }}=R_{\infty}+\frac{R_{0}-R_{\infty}}{1+(j \omega \tau)^{\alpha}}
$$

In order to make a comparison between the different cell lines, the dimensions of the cells (major (D1) and minor (D2) axes) were first measured. From these two dimensions, the average cell diameter, defined as the mean value of the major axis and the minor axis, was calculated. In Fig. 2c (lower insert) the central relaxation frequency $\left(\mathrm{f}_{\mathrm{c}}\right)$ extracted from the Cole model fitting is plotted against the measured average cell 
diameter D. As observed, there is a good correlation between the cell diameter and the characteristic frequency showing an inversely proportional relation.

In the proposed analysis, the differences obtained in $\mathrm{f}_{\mathrm{c}}$ can be mainly attributed to the cell size and organization of the cell monolayers. For this reason, the values obtained for this parameter in the case of $\mathrm{C} 2 \mathrm{C} 12$ and HeLa cells are very similar as they are also very similar in size and cell monolayer organization. On the contrary, the system is able to show a clear difference in the DC-3F cell line, whose organization and cell size also differs clearly from the other two cell lines.

The goal in this section was to test the ability of the system to extract differential characteristics of the cell lines under study. These results show the ability of the system to extract information of the preelectroporation state of the cell monolayer under treatment in a non-invasive/non-destructive way. Due to the specific spiral electrodes topology used, which covers almost the whole surface of each culture well, the measurements provide an average information about the characteristics of the cell monolayer. Contrarily, most of the microelectrodes structures usually used for registering the impedance of cell monolayers provide only a local measurement, thus being highly dependent on the state of the cells in these specific measuring points. The information about the cell monolayers before electroporation could be useful to assess the state of the sample before treatment and it could also be useful for optimizing the parameters of the subsequent electroporation treatment. 
a)
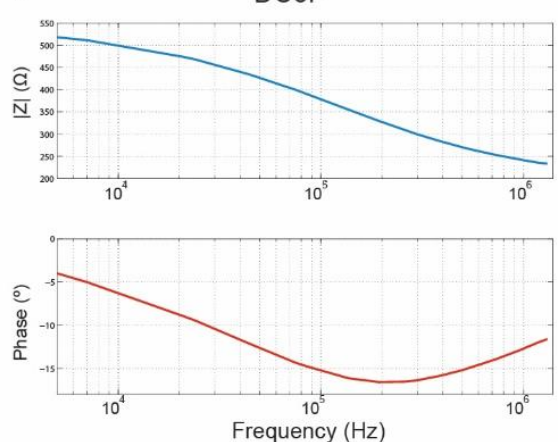

b)
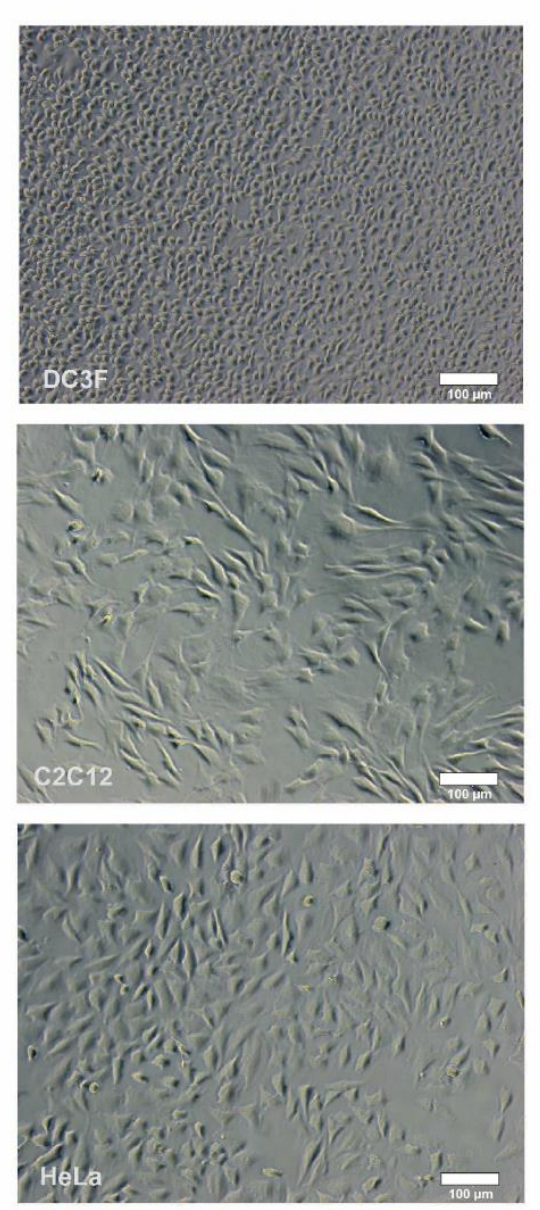

$\mathrm{C} 2 \mathrm{C} 12$
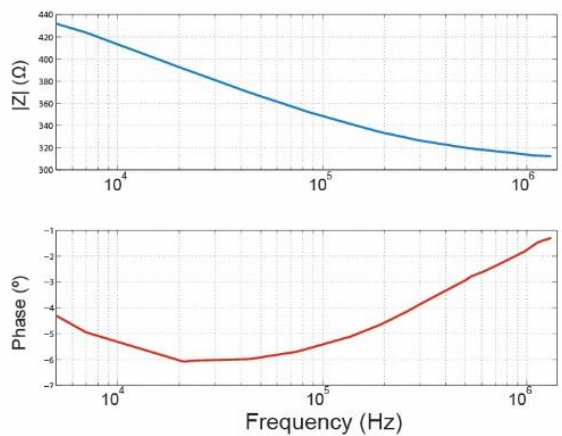

c)
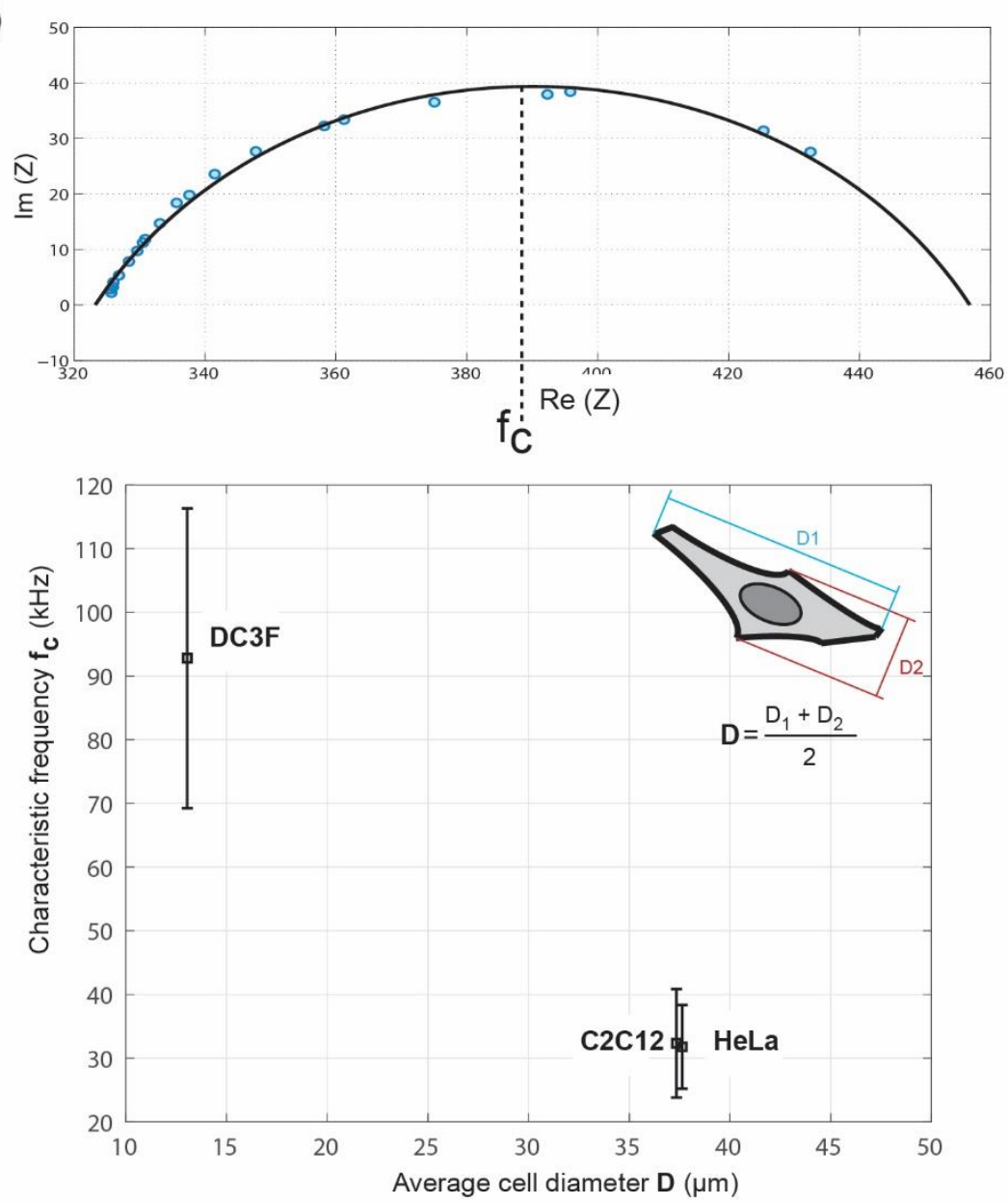

Fig.2. Pre-electroporation impedance measurements. a) Representative example of the impedance spectra (magnitude and phase) prior to electroporation application. b) Representative brightfield micrographs of the three cell lines under study after pulse application (10x magnification). c) Upper insert: example of Nyquist plot for the impedance spectrum of the $\mathrm{C} 2 \mathrm{C} 12$ cells (blue dots) and its corresponding Cole model after fitting (solid line). Lower insert: average cell diameter VS mean characteristic frequency parameter (fc) obtaiend from the Cole model fitting for the three cell lines under analysis. 


\subsection{Interpulse measurements}

In this section the impedance measurements performed between consecutive pulses are shown. As explained in the Materials and Methods section, 860 spectra ( 1 spectrum/ms) were continuously acquired in the time gap ( 1 second) between two consecutive pulses. In Fig. 3a an example of the magnitude and phase evolution of a complete 8-pulses electroporation procedure is shown for the $\mathrm{C} 2 \mathrm{C} 12$ cells and an electric field intensity of $1000 \mathrm{~V} / \mathrm{cm}$. The response of Hela cells to the same pulsing conditions is very similar (see supplementary Fig. A for more details). A reduction in both impedance magnitude and phase is observed along the spectra shown in the Bode plots. Pulse after pulse the spectra becomes flatter in accordance with a permeabilization of the cell membrane. In the frequency band studied, the porated membrane no longer behaves as an insulator because the membrane is partially shunted by a transmembrane resistor (the permeabilization structures), thus reducing its impedance. In the case of the impedance magnitude, as expected, cell permeabilization has a more significant impact in the lower frequencies studied. At the higher frequencies the impact becomes less and less pronounced. In the case of the phase angle, the most pronounced variation corresponds to the middle frequencies studied. It is around these middle frequencies where the phase displays its minimum value corresponding to the dielectric relaxation of biological tissues caused by the presence of membranes ( $\beta$ dispersion). It is thus reasonable to think that if electroporation is preferentially affecting the membrane of the cells, the phase around these frequencies should be highly impacted.

The evolution over time at each individual frequency of both magnitude and phase is represented in Fig. $3 b$ for the same data previously shown. From these plots it is obvious to conclude that the dynamic changes of impedance vary with the measured frequency. It can be observed how at low frequencies two different dynamics are observable: a fast change after each pulse and a continuous decrease over time. At high frequencies, only a slow single trend is observed. In Fig. 3c this evolution is detailed for two specific frequencies: for the magnitude, $7 \mathrm{kHz}$ and $1.12 \mathrm{MHz}$, corresponding to the low and high frequency bands respectively, and for the phase, the middle frequency band $(75 \mathrm{kHz})$ and the high frequency band $(1.12$ $\mathrm{MHz}$ ). The observed fast recovery of impedance immediately after each pulse application can be explained by the short-term membrane resealing between pulses. Secondly, an accumulated impedance reduction with a slower dynamical behavior is superimposed to the fast changes. This second persistent decrease in impedance can be justified at least by two different phenomena. First, as it could be expected, by accumulated membrane damage over the electroporation procedure that causes the decrease of the impedance. According to electroporation classical data, perturbation in the cell membrane can last from minutes to hours. However, a second phenomenon could be responsible for the observed slow impedance 
decrease. This is the leakage of ions, mostly potassium, from the cell cytosol to the extracellular buffer. Under the particular experimental conditions used in these measurements, the low conductivity of the electroporation buffer used could be modified by the transport of ions from the cell inside to the extracellular space. This would increase the extracellular conductivity, which translates in a reduction of the measured impedance.

Observing now the evolution at high frequency, a slow linear reduction of impedance over time is detected. In an ideal situation, with no other collateral effect apart from membrane permeabilization, the impedance at high enough frequencies should not be affected by electroporation. Consequently, the reduction observed at these high frequencies must be necessarily the result of a collateral effect during cell permeabilization. The mentioned change in conductivity produced by the leakage of ions is the most plausible explanation and could follow this linear behavior. Other phenomena associated with cell electroporation could also be responsible of this effect. Among them, cell swelling, due to water penetration in the cell facilitated by membrane permeabilization, could impact the overall measured impedance. However, the dynamics of this change are in the order of minutes and not seconds. The absence of cell swelling was confirmed after visualizing the cells under bright field microscopy during the first 5 minutes after the pulses application. Finally, an increase in temperature due to Joule heating could also produce a reduction in impedance. However, the accumulated temperature change was calculated and resulted to be negligible to explain the observed change $\left(\approx 0.3{ }^{\circ} \mathrm{C}\right.$ for 8 pulses of $100 \mu$ s at $\left.1400 \mathrm{~V} / \mathrm{cm}\right)$. 


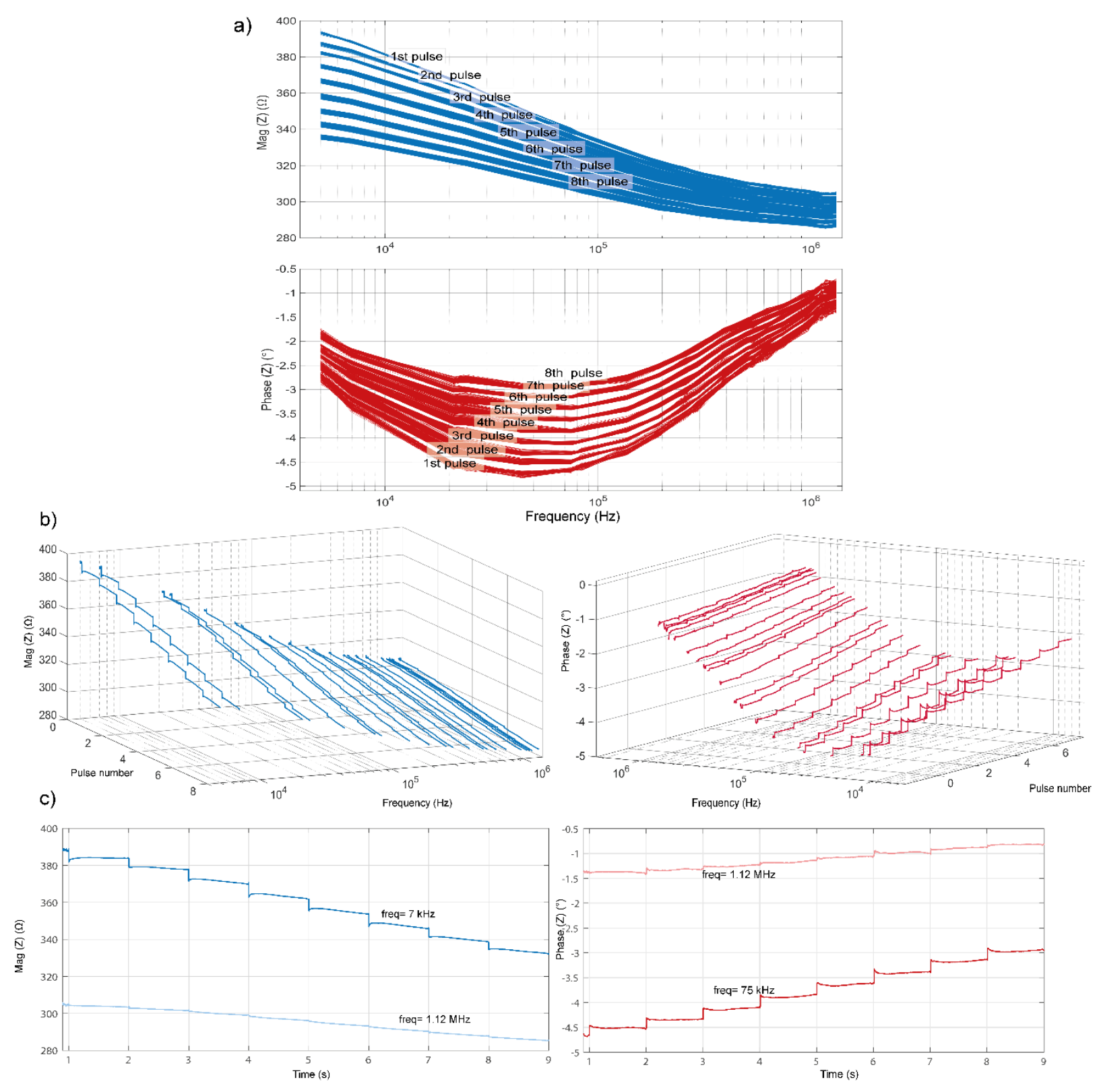

Fig. 3. Impedance spectrum evolution during electroporation. Data shown corresponds to a representative example for $\mathrm{C} 2 \mathrm{C} 12$ cells exposed to an electric field intensity of $1000 \mathrm{~V} / \mathrm{cm}$. a) Bode plots showing the evolution in the impedance spectrum (magnitude and phase) pulse after pulse for a complete 8 pulses exposure experiment. b) 3D plot for the temporal evolution of the impedance magnitude and phase evolution at each individual frequency measured. c) Detailed temporal evolution in the impedance at two specific frequencies: $7 \mathrm{kHz}$ and $1.12 \mathrm{MHz}$ for the magnitude, and $75 \mathrm{kHz}$ and $1.12 \mathrm{MHz}$ for the phase. 
The same set of data is shown in this case for the DC-3F cell line in Fig. 4. Similar to the previous case, the evolution of the Bode plots during the complete 8-pulses exposure depicted in Fig. 4a. shows changes in the impedance spectra specially affecting the impedance magnitude at the low frequencies and the phase spectrum at intermediate frequencies. These results are in accordance with the previous explanation given above for Fig. 3a.

The dynamic temporal evolution for the DC-3F cell line at two different frequencies is shown in Fig. 4b both for magnitude (at $7 \mathrm{kHz}$ and $1.12 \mathrm{MHz}$ ) and phase (at $137 \mathrm{kHz}$ and $1.12 \mathrm{MHz}$ ). An overall similar behavior compared to $\mathrm{C} 2 \mathrm{C} 12$ and Hela cells can be observed. However, the evolution in the magnitude at low frequency (Fig. 4b left) reveals a different behavior. The fast recovery of the magnitude immediately after each pulse, which is clearly noticeable for the two previous cell lines, is not evident and seems to be masked by a dominating slow impedance decay accumulated along the complete measuring time, likely due to the ion diffusion. The different behavior of this cell line can be at least partially explained based on the size (smaller) and thus, the number (higher) of cells exposed (refer to Fig. 2b left panel). This difference would be translated in a much larger diffusion of ions to the extracellular media, producing a considerably higher change in conductivity that corrupts the measurements. On the contrary, if the phase evolution is now evaluated, it can be observed how it still displays the fast recovery pulse after pulse similar to the other two cell lines. This observation suggests that if the effect of conductivity changes in the extracellular bulk electrolyte is the responsible for this masking effect in the impedance magnitude, this has considerably less impact in the phase angle of impedance. The similarities observed in the behavior of phase angle for the different cell lines reinforce the fact that phase is more immune to conductivity variations and represents a more appropriate parameter for comparison. 

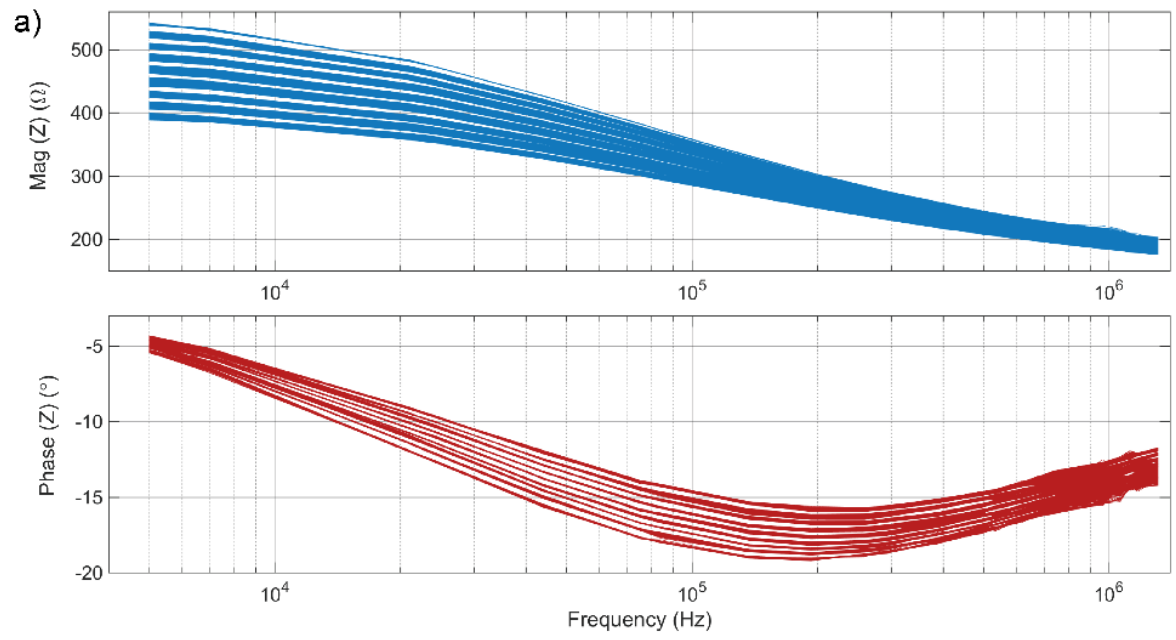

b)
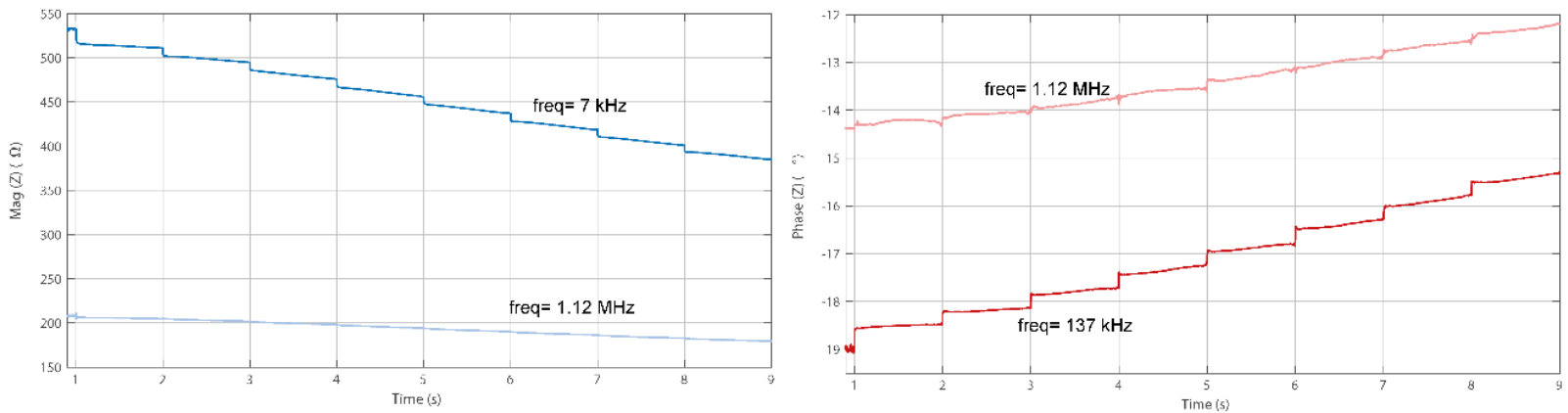

Fig. 4. Impedance spectrum evolution for a representative example for DC-3F cells exposed to an electric field intensity of $1000 \mathrm{~V} / \mathrm{cm}$. a) Bode plots showing the evolution in the impedance spectrum (magnitude and phase) pulse after pulse for a complete 8 pulses exposure experiment. b) Detailed temporal evolution in the impedance at two specific frequencies: $7 \mathrm{kHz}$ and $1.12 \mathrm{MHz}$ for the magnitude, and $137 \mathrm{kHz}$ and $1.12 \mathrm{MHz}$ for the phase. 


\subsection{Modeling}

In order to better extract all the information provided by the multifrequency impedance measurements, an electrical equivalent model was used in this study. The goal was trying to separate the contributions of the impedance change, produced by the cell membrane electroporation itself, and the collateral change in impedance likely caused by the diffusion of ions, observed in the high frequency measurements. The model includes a resistive component $\left(R_{\text {pores }}\right)$ corresponding to the changes produced in the membrane by electroporation and another resistive component in the extracellular space $\left(\mathrm{R}_{\text {ext }}\right)$ taking into account the changes in conductivity produced in the extracellular buffer. Additionally, a constant phase element $\left(\mathrm{CPE}_{\mathrm{m}}\right)$, in parallel with $\mathrm{R}_{\text {pores}}$, models the capacitive behavior of the membrane, and finally, another resistance is introduced for the intracellular space $\left(\mathrm{R}_{\text {int }}\right)$. In Fig. 5a a schematic representation of the model with the different components is shown. The multifrequency impedance data for each cell line corresponding to measurements during exposure of cells to three different electric field intensities (600, 1000 and $1400 \mathrm{~V} / \mathrm{cm}$, respectively) were fitted to the model in MATLAB. In the case of the C2C12 and Hela cells the only restriction imposed to the fitting algorithm was a lower boundary to the parameter $\mathrm{R}_{\text {int }}$ corresponding to its initial value. Under the considered experimental conditions this parameter is expected not to decrease during the electroporation procedure. The rest of parameters were left to freely vary during the fitting process.

In Fig. $5 b$ the evolution of both $R_{\text {pores }}$ and $R_{\text {ext }}$, normalized with respect to their initial values, is shown for representative examples of the three electric field intensities assayed in $\mathrm{C} 2 \mathrm{C} 12$ and Hela cells. The results show how the parameter $\mathrm{R}_{\text {pores }}$ displays an abrupt decrease followed by a fast recovery after each pulse application. This behavior is compatible with membrane permeabilization occurring during the pulse and a fast membrane resealing in the interval between pulses. While the amplitude of the abrupt decrease after each pulse is almost constant over time for the low electric field intensity, for the higher intensity, it decreases with time. This is compatible with a possible loss of efficacy (saturation effect) of the repetitive pulse application for conditions where the cell membranes are extensively permeabilized. As observed, in the interval between consecutive pulses, $\mathrm{R}_{\text {pores }}$ does not recover to its initial level, what produces an accumulated decrease of this parameter over time. As explained in the previous section, this decrease is compatible with an accumulated membrane damage over the electroporation procedure. The parameter $R_{\text {ext }}$ displays a monotonous decrease over time highly dependent on the intensity of the electric field applied and compatible with a diffusion of ions from the cell cytosol to the extracellular buffer. 
The results given by the model are in agreement with the previous interpretation of the impedance magnitude and phase information. The model confirms that the observed continuous decrease in the impedance at high frequencies is compatible with an undesired change in conductivity of the extracellular medium reflected in the parameter $R_{\text {ext }}$. By combining the whole acquired spectral information in the model, it is possible to isolate and separate this undesired effect to have a better access to the fast events occurring in the cell membrane during electroporation. The fast recovery of membrane after each pulse is no longer masked by the collateral change in conductivity.

Finally, in the case of the DC-3F cell line, the fitting boundary conditions used for the other two cell lines produced non stable results. In order to study the evolution in the parameter $\mathrm{R}_{\mathrm{pores}}$, which reflects the effects of electroporation, the behavior of $\mathrm{R}_{\mathrm{ext}}$ was fixed to the model as an input taken in the following way: the behavior of $R_{\text {ext }}$ was extracted from the dynamics of the impedance at high frequency, which, as previously discussed, should correspond to the buffer conductivity changes; thus, the evolution of the real part of the impedance at high frequency ( $\mathrm{f}=1.12 \mathrm{MHz}$ ) was extracted, normalized and introduced into the model. Fig. $5 c$ (right panel) shows the imposed variation in $R_{\text {ext }}$ for the different electric field intensities assayed. It is observed how this parameter displays a very similar behavior compared to the $\mathrm{R}_{\mathrm{ext}}$ automatically obtained from the model for the other two cell lines, what supports the validity of this approach.

The results in Fig. 5c (left panel) show how the obtained parameter $R_{\text {pores }}$ displays, similarly to the other two cell lines, a double dynamic evolution. However, the fast recovery after each pulse seems to be still affected by a constant decrease in the impedance for the two highest electric field intensities. This behavior is difficult to interpret and would mean that cell membranes continue to open in the interval between electric pulses what is very unlikely. This suggests that, even under these fitting conditions, if the change in conductivity produced during the electroporation pulses is very high, the model is unable to completely capture and isolate the membrane resealing dynamics in the parameter $\mathrm{R}_{\text {pores. }}$. 
a)
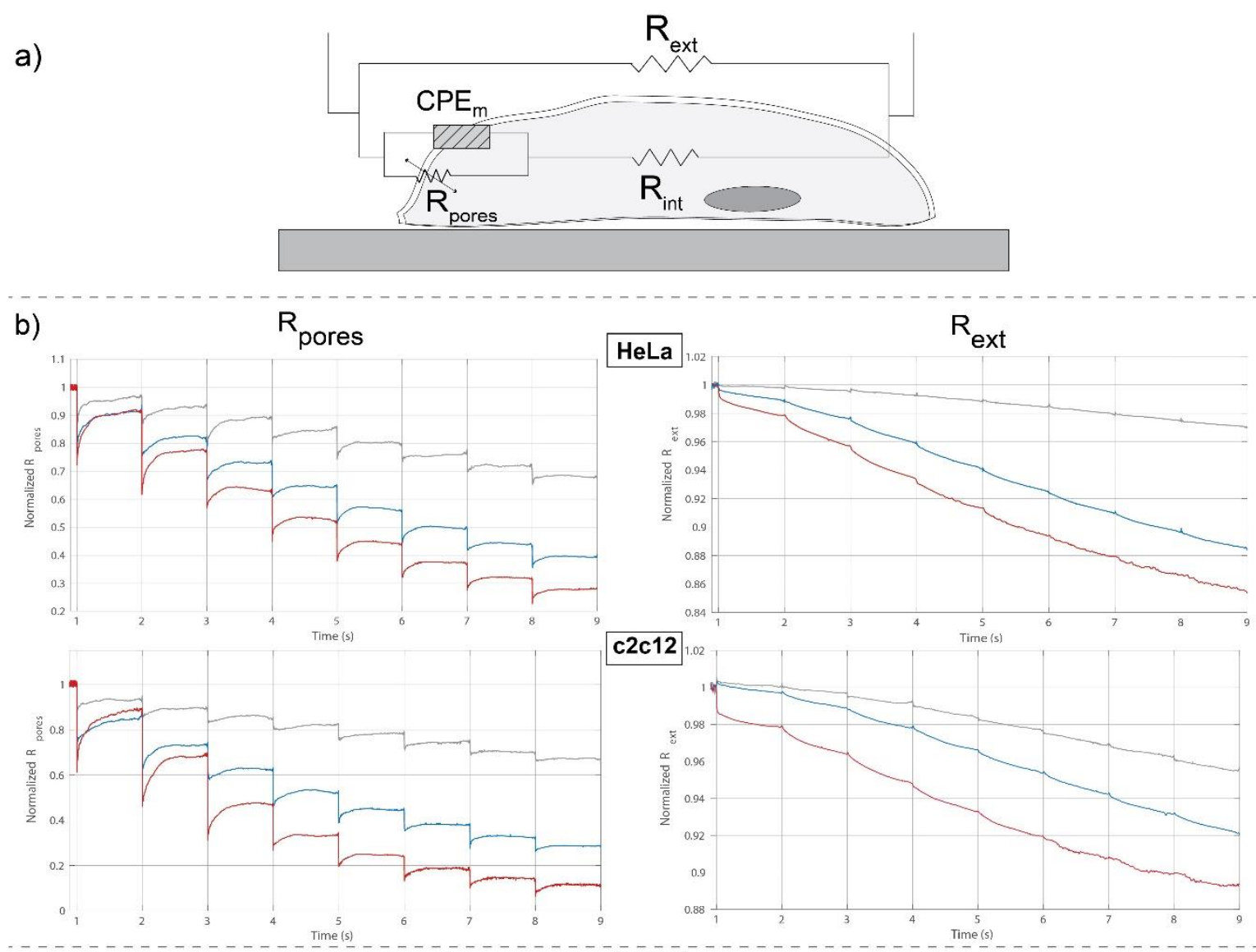

c)

DC-3F

Fixed $R_{\text {ext }}$


$-600 \mathrm{~V} / \mathrm{cm} \quad-1000 \mathrm{~V} / \mathrm{cm} \quad-1400 \mathrm{~V} / \mathrm{cm}$

Fig. 5. Electrical equivalent modelling results. a) Schematic representation of the equivalent circuit model studied. b) Evolution of the model parameters $\mathrm{R}_{\text {pores }}$ and $\mathrm{R}_{\text {ext }}$ for Hela and $\mathrm{C} 2 \mathrm{C} 12$ cells normalized to their initial values. A representative example of the three electric field intensities assayed is shown (see color legend at the bottom of the figure). c) Evolution of the model parameter $\mathrm{R}_{\text {pores }}$ obtained for DC-3F cells and the imposed evolution for $\mathrm{R}_{\mathrm{ext}}$ included in the fitting algorithm. Notice that axes are not to scale. 


\subsection{Comparative analysis with fluorescence microscopy}

In this section the goal is to compare the information extracted from the impedance measurements to the success of membrane permeabilization studied by the internalization of the fluorescent dye YoPro-1, which is commonly used in electroporation experiments. In order to perform this comparison, the impedance decay accumulated during the complete electroporation treatment was used. As discussed above, the accumulated decrease over time observed at low frequency can be explained by two different processes occurring simultaneously: first, the progressive increase in membrane permeability as a consequence of the accumulation of membrane defects caused by the consecutive electric pulses and second, a change in conductivity due to the diffusion of ions. As shown by the model in the previous section, the conductivity variations can be approached from the impedance changes at high frequency. Consequently, by combining the low and high frequency information, the undesired change in conductivity can be, at least partially compensated in a simple way, which allows the study of the decrease in impedance due to membrane poration in a separate manner.

In Fig. 6a an example of the information used to calculate the total impedance change during the complete electroporation treatment is shown for $\mathrm{C} 2 \mathrm{C} 12$ cells pulsed with an electric field intensity of $1400 \mathrm{~V} / \mathrm{cm}$. The long term evolution in the impedance magnitude at the lowest and the highest frequencies was extracted and subsequently, the quotient between these two quantities was calculated and normalized to show data in percentage of total change with respect to the initial (pre-electroporation) state. Fig. 6b shows the data for the resulting mean long-term impedance decay for all cell lines and electric field intensities tested (data correspond to the mean of at least three separate repetitions for each electric field intensity assayed). In all cases, it can be clearly noticed an electric field dependence with an increasing percentage of variation related to the increasing electric field intensity. It is also noticed that for the highest electric field intensity, there is a change from a linear decrease with pulse number to an asymptotic evolution at the end of the treatment. This observation suggests a reduction in the effectiveness of electroporation with pulse number in accordance with the concept of cell electro-desensitization (Silve et al., 2014). As suggested by the measurements, when the state of accumulated membrane perturbation is very high, the electric field would be less effective in the creation of new permeable structures leading to a saturation effect. 


\section{a)}
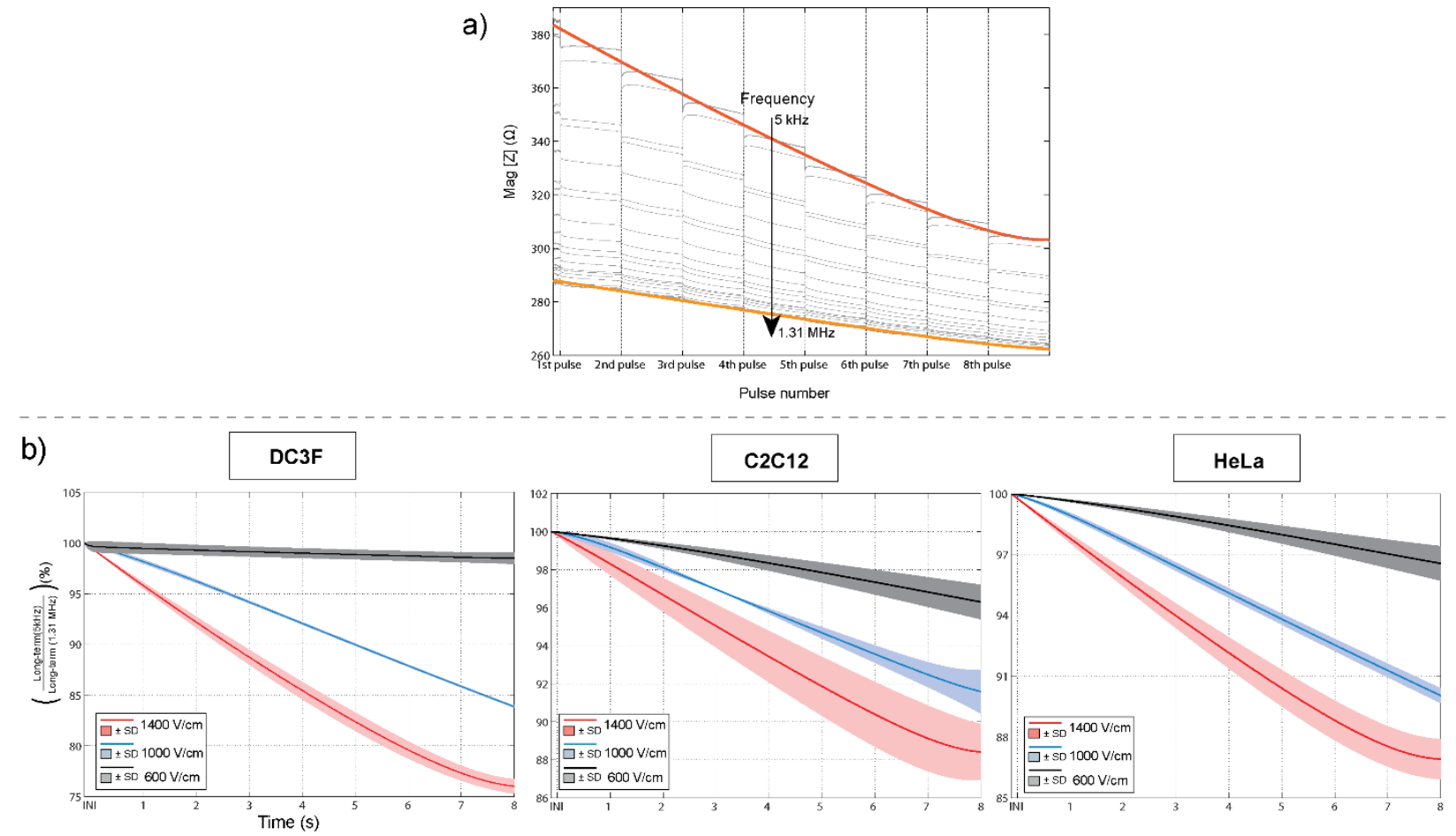

Fig. 6. Slow impedance variations. a) Example of the information extracted to calculate the total impedance change during the complete electroporation treatment. Low and high impedance are highlighted for a condition where $\mathrm{C} 2 \mathrm{C} 12$ cells were pulsed with an electric field intensity of $1400 \mathrm{~V} / \mathrm{cm}$. b) Mean impedance evolution calculated as the ratio between low frequency $(5 \mathrm{kHz})$ and high frequency $(1.31 \mathrm{MHz})$ impedance magnitude normalized in $100 \%$ to the initial pre-electroporation values for the three cell lines under analysis. Mean values \pm standard deviation $(\mathrm{SD})$ were calculated from at least triplicates for each electric field intensity assayed. 
Once the impedance information was analyzed the goal was to study if this information could be useful as an indicator of the success of the treatment compared to a traditional technique based on the use of a fluorescent dye. Fig. 7 (right panels) shows, as an example, a fluorescent micrograph of each cell line after treatment for the maximum electric field assayed. It can be noticed that for this condition almost $100 \%$ of cells were permeabilized in all cases. It is noticeable that in the case of DC3F cells, due to the small size of this cell line, it is possible to observe black areas (non permeabilized) corresponding to the region under the microelectrodes where the electric field is below the permeabilization threshold, which is high in the case of the DC-3F cells because of their very little size. In order to quantify the extent of permeabilization, at least three different fields of each sample were recorded under the fluorescence microscope and subsequently automatically counted with the free image processing software ImageJ (MacBiophotonics). The mean number of cells obtained for the maximum electric field was considered as $100 \%$ permeabilization and the rest of results normalized with respect to this value.

Following, the total $\%$ of impedance variation extracted from the value at the end of the treatment $(\mathrm{t}=8 \mathrm{~s})$ (see Fig. 6) was compared with the obtained permeabilization rates. In Fig. 7 (left panels) both values are plotted together (notice that the scales in both axes are not the same). The results in Fig. X show a clear correlation between impedance changes and permeabilization rates for the three cell lines analyzed. Most interestingly, the statistical analysis shows that, in the case of $\mathrm{C} 2 \mathrm{C} 12$ and HeLa cells, the differences obtained in the permeabilization rates for $1000 \mathrm{~V} / \mathrm{cm}$ and $1400 \mathrm{~V} / \mathrm{cm}$ calculated using a fluorescent dye were not significant. However, for the same conditions, the average impedance variation differed significantly. This result can be explained based on the fact that the quantification of Yo-Pro-1 uptake is an average effect captured several minutes after pulse. However, in the case of impedance measurements the information is recorded in real time during pulse application. Thus, the long-term uptake of Yo-Pro-1 after the application of $1000 \mathrm{~V} / \mathrm{cm}$ or $1400 \mathrm{~V} / \mathrm{cm}$ can be similar while the effect of pulses on cells is not the same. This observation demonstrates how electrical impedance can quantitatively measure the effects produced in the cell membrane for different electric fields better than traditional permeabilization tests due to the possibility of recording in real time. 

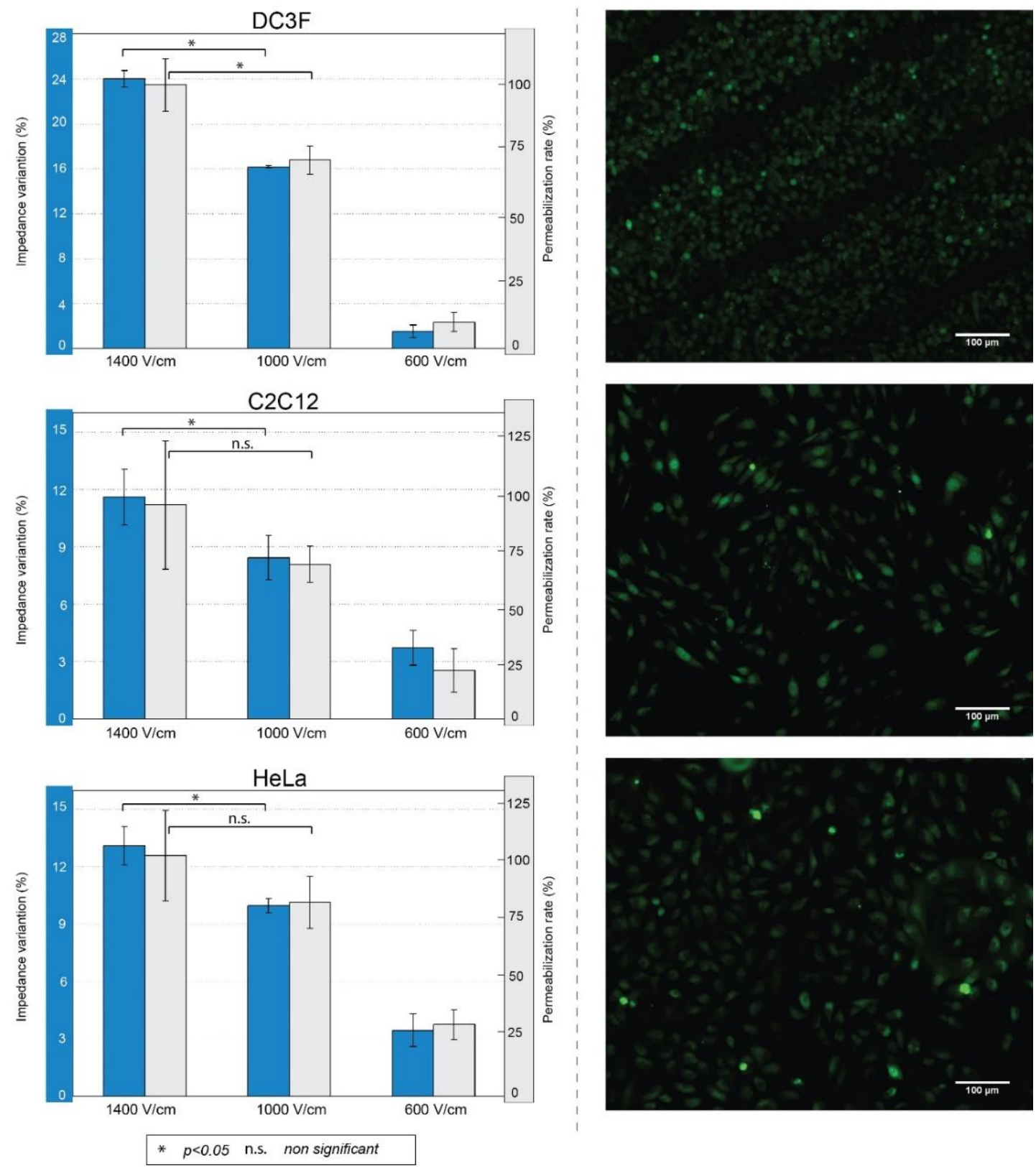

Fig. 7. Fluorescence versus impedance quantification. Right panels) an example of a fluorescent micrograph of each cell line after treatment with 8 electric pulses of $1400 \mathrm{~V} / \mathrm{cm}$ is shown (10x magnification). Left panels) Comparison between the total \% of impedance variation (in blue) extracted from the value at the end of the treatment $(t=8 \mathrm{~s})$ and the quantified permeabilization rates using fluorescence dye Yo-Pro1 (in grey). The mean number of fluorescent cells obtained for the maximum electric field was considered as the $100 \%$ and the rest of the results were normalized with respect to this value. Notice that the scales in both axes are not the same. 


\section{Conclusions}

In this study the evolution of the electrical impedance during electroporation of three cell lines was analyzed and compared. The utilized setup allowed to acquire electrical impedance spectroscopy measurements of the cell monolayers under study growing in standard multiwell plates in a minimally invasive way. First, the impedance spectra of the different cell lines prior to electroporation was analyzed using the Cole model. The results showed how the system is able to detect different impedance spectra based on the cell line under analysis demonstrating the sensitivity of the present setup to the differential characteristics of a cell monolayer. Specifically, the obtained characteristic frequency parameter $\left(f_{c}\right)$ from the Cole model was correlated with the average cell diameter showing an inverse relation. $\mathrm{C} 2 \mathrm{C} 12$ myoblasts and HeLa cells, which are very similar in size, displayed also very similar values of $f_{c}$ while DC-3F cells displayed a shift in the value of this parameter consequent with the smallest size of these cells.

Subsequently, the impedance response during electroporation was analyzed in the interval between consecutive pulses thanks to the fast measuring approach based on multisine excitations used. This measuring strategy allowed to obtain one complete impedance spectrum $(5 \mathrm{kHz}-1.3 \mathrm{MHz})$ per millisecond, thus enabling to capture the fast dynamics in the impedance variations during the electroporation protocol. The analysis of the magnitude and phase angle of the impedance revealed two differentiated behaviors of the impedance. While at the lower/middle frequencies the impedance displayed a fast recovery after each pulse likely corresponding to the membrane resealing, at the higher frequencies only a monotonous impedance decrease was observed. A change in conductivity of the extracellular buffer due to ion diffusion from the cell cytoplasm through the permeabilized cell membrane could explain this second effect. The measurements show how the impedance magnitude is more affected by this conductivity change than the phase angle. For this reason, in the case of the phase angle it is possible to observe the fast impedance recovery immediately after pulsing for all cell lines while in the case of the magnitude it appears to be masked in some cases. This leads to conclude that the phase angle is more suitable and immune to undesired effects for the observation of the fast impedance variations in electroporation.

In order to maximize the extracted information from the multifrequency impedance spectrum, an electrical equivalent circuit was used. This model represented a good option to give a theoretical framework in the study of the contribution of the different effects to the system. The results obtained by the model confirmed the hypothesis about the impact that conductivity changes in the extracellular buffer had in the measurements and could give explanation to the observed high frequency variations. Thanks to the separation of the different contributions, the fast dynamics in the membrane could be better observed. 
However, the model did not always give satisfactory results in all situations and needed some a-priori information for the proper running of the fitting algorithm. This makes this analysis method more suitable for theoretical studies or as a tool for performing a preliminary study of a particular measuring setup. However, for practical applications, for example in real time control loops based on impedance measurements, it seems reasonable to use faster and simpler approaches. based, for example, in twofrequency measurements.

In this study, the prior use of the model enabled us to understand the observed contribution at high frequency and subsequently to extract it from the low frequency information in a simple way. The use of the high frequency information to compensate the collateral effects produced during electroporation represented an advantage in front of using single frequency measurements. This enabled us to perform a fast and simple quantification of the impedance variation with the applied electric and to compare it to the permeabilization rates obtained using a classical fluorescent dye. The results from this comparison showed a perfect match between impedance data and fluorescent information for the three cell lines under analysis. Most interestingly, impedance information provided statistical significance for cases where the fluorescent information was not significant. This observation demonstrates how electrical impedance can quantitatively measure the effects produced in the cell membrane for different electric field intensities more accurately than traditional permeabilization tests due to the possibility of recording in real time. 


\section{References:}

Andre, F., Mir, L.M., 2004. DNA electrotransfer: its principles and an updated review of its therapeutic applications. Gene Ther 11 Suppl 1, S3342. doi:10.1038/sj.gt.3302367

Andrei G Pakhomov and Marko S Markov, D.M., Andrei G Pakhomov and Marko S Markov, D.M., 2010. Advanced Electroporation Techniques in Biology and Medicine. CRC Press.

Batista Napotnik, T., Miklavčič, D., 2018. In vitro electroporation detection methods - An overview. Bioelectrochemistry 120, $166-182$. doi:10.1016/j.bioelechem.2017.12.005

Castellví, Q., Mercadal, B., Ivorra, A., 2017. Assessment of Electroporation by Electrical Impedance Methods, in: Miklavčič, D. (Ed.), Handbook of Electroporation. Springer International Publishing, Cham, pp. 671-690. doi:10.1007/978-3-319-32886-7_164

Cukjati, D., Batiuskaite, D., Andre, F., Miklavcic, D., Mir, L.M., 2007. Real time electroporation control for accurate and safe in vivo non-viral gene therapy. Bioelectrochemistry 70, 501-507. doi:S1567-5394(06)00162-9 [pii] 10.1016/j.bioelechem.2006.11.001

Davalos, R. V, Otten, D.M., Mir, L.M., Rubinsky, B., 2004. Electrical impedance tomography for imaging tissue electroporation. IEEE Trans Biomed Eng 51, 761-767. doi:10.1109/TBME.2004.824148

Foster, K.R., Schwan, H.P., 1989. Dielectric properties of tissues and biological materials: a critical review. Crit Rev Biomed Eng 17, $25-104$.

García-Sánchez, T., Azan, A., Leray, I., Rosell-Ferrer, J., Bragós, R., Mir, L.M., 2015. Interpulse multifrequency electrical impedance measurements during electroporation of adherent differentiated myotubes. Bioelectrochemistry 105. doi:10.1016/j.bioelechem.2015.05.018

García-Sánchez, T., Guitart, M., Rosell-Ferrer, J., Gómez-Foix, A., Bragós, R., 2014. A new spiral microelectrode assembly for electroporation and impedance measurements of adherent cell monolayers. Biomed. Microdevices 16, 575-590. doi:10.1007/s10544-014-9860-6

García-Sánchez, T., Guitart, M., Rosell-Ferrer, J., Gómez-Foix, A.M., Bragós, R., 2014. A new spiral microelectrode assembly for electroporation and impedance measurements of adherent cell monolayers. Biomed. Microdevices 16. doi:10.1007/s10544-014-9860-6

Garner, A.L., Nianyong, C., Jing, Y., Kolb, J., Swanson, R.J., Loftin, K.C., Beebe, S.J., Joshi, R.P., Schoenbach, K.H., 2004. Time domain dielectric spectroscopy measurements of HL-60 cell suspensions after microsecond and nanosecond electrical pulses. Plasma Sci. IEEE Trans. 32, 2073-2084. doi:10.1109/tps.2004.835973

Ivorra, A., Al-Sakere, B., Rubinsky, B., Mir, L.M., 2009. In vivo electrical conductivity measurements during and after tumor electroporation: conductivity changes reflect the treatment outcome. Phys Med Biol 54, 5949-5963. doi:S0031-9155(09)20674-9 [pii]10.1088/00319155/54/19/019

Ivorra, A., Rubinsky, B., 2007. In vivo electrical impedance measurements during and after electroporation of rat liver. Bioelectrochemistry 70 , 287-295. doi:S1567-5394(06)00147-2 [pii]10.1016/j.bioelechem.2006.10.005

Kennedy, S.M., Ji, Z., Hedstrom, J.C., Booske, J.H., Hagness, S.C., 2008. Quantification of electroporative uptake kinetics and electric field heterogeneity effects in cells. Biophys. J. 94, 5018-5027. doi:10.1529/biophysj.106.103218

Khine, M., Ionescu-Zanetti, C., Blatz, A., Wang, L.-P., Lee, L.P., 2007. Single-cell electroporation arrays with real-time monitoring and feedback control. Lab Chip 7, 457-462.

Kinosita Jr, K., Tsong, T.Y., 1979. Voltage-induced conductance in human erythrocyte membranes. Biochim. Biophys. Acta - Biomembr. 554, 479-497. doi:http://dx.doi.org/10.1016/0005-2736(79)90386-9

Martinsen, S.G. and O.G., 2000. Bioimpedance and Bioelectricity Basics. Academic Press, San Diego, CA.

Marty, M., Sersa, G., Garbay, J.R., Gehl, J., Collins, C.G., Snoj, M., Billard, V., Geertsen, P.F., Larkin, J.O., Miklavcic, D., Pavlovic, I., PaulinKosir, S.M., Cemazar, M., Morsli, N., Soden, D.M., Rudolf, Z., Robert, C., O’Sullivan, G.C., Mir, L.M., 2006. Electrochemotherapy - An easy, highly effective and safe treatment of cutaneous and subcutaneous metastases: Results of ESOPE (European Standard Operating Procedures of Electrochemotherapy) study. Eur. J. Cancer, Suppl. 4, 3-13. doi:10.1016/j.ejcsup.2006.08.002

Mir, L.M., Orlowski, S., Belehradek Jr., J., Paoletti, C., 1991. Electrochemotherapy potentiation of antitumour effect of bleomycin by local electric pulses. Eur J Cancer 27, 68-72.

O’Neill, R.J., Tung, L., 1991. Cell-attached patch clamp study of the electropermeabilization of amphibian cardiac cells. Biophys. J. 59, 10281039. doi:http://dx.doi.org/10.1016/S0006-3495(91)82318-9

Pasqualotto, E., Ferrario, A., Scaramuzza, M., De Toni, A., Maschietto, M., 2012. Monitoring Electropermeabilization of Adherent Mammalian Cells Through Electrochemical Impedance Spectroscopy. Procedia Chem. 6, 79-88. doi:http://dx.doi.org/10.1016/j.proche.2012.10.133 
Pavlin, M., Leben, V., Miklavčič, D., 2007. Electroporation in dense cell suspension-Theoretical and experimental analysis of ion diffusion and cell permeabilization. Biochim. Biophys. Acta - Gen. Subj. 1770, 12-23. doi:http://dx.doi.org/10.1016/j.bbagen.2006.06.014

Pliquett, U., Langer, R., Weaver, J.C., 1995. Changes in the passive electrical properties of human stratum corneum due to electroporation. Biochim. Biophys. Acta - Biomembr. 1239, 111-121. doi:http://dx.doi.org/10.1016/0005-2736(95)00139-T

Pliquett, U.F., Schoenbach, K.H., 2009. Changes in electrical impedance of biological matter due to the application of ultrashort high voltage pulses. Dielectr. Electr. Insul. IEEE Trans. 16, 1273-1279. doi:10.1109/tdei.2009.5293938

Pucihar, G., Kotnik, T., Miklavčič, D., Teissié, J., 2008. Kinetics of Transmembrane Transport of Small Molecules into Electropermeabilized Cells. Biophys. J. 95, 2837-2848. doi:http://dx.doi.org/10.1529/biophysj.108.135541

Romeo, S., Wu, Y.H., Levine, Z.A., Gundersen, M.A., Vernier, P.T., 2013. Water influx and cell swelling after nanosecond electropermeabilization. Biochim. Biophys. Acta - Biomembr. 1828, 1715-1722. doi:10.1016/j.bbamem.2013.03.007

Schwan, H.P., 1994. Electrical properties of tissues and cell suspensions: mechanisms and models, in: Engineering in Medicine and Biology Society, 1994. Engineering Advances: New Opportunities for Biomedical Engineers. Proceedings of the 16th Annual International Conference of the IEEE. pp. A70-A71 vol.1. doi:10.1109/iembs.1994.412155

Silve, A., Guimerà Brunet, A., Al-Sakere, B., Ivorra, A., Mir, L.M., 2014. Comparison of the effects of the repetition rate between microsecond and nanosecond pulses: Electropermeabilization-induced electro-desensitization? Biochim. Biophys. Acta - Gen. Subj. 1840, $2139-2151$. doi:http://dx.doi.org/10.1016/j.bbagen.2014.02.011

Stolwijk, J.A., Hartmann, C., Balani, P., Albermann, S., Keese, C.R., Giaever, I., Wegener, J., 2011. Impedance analysis of adherent cells after in situ electroporation: Non-invasive monitoring during intracellular manipulations. Biosens. Bioelectron. 26, 4720-4727. doi:http://dx.doi.org/10.1016/j.bios.2011.05.033

Wegner, L.H., Frey, W., Schönwälder, S., 2013. A critical evaluation of whole cell patch clamp studies on electroporation using the voltage sensitive dye ANNINE-6. Bioelectrochemistry 92, 42-46. doi:http://dx.doi.org/10.1016/j.bioelechem.2013.03.002

Yarmush, M.L., Golberg, A., Serša, G., Kotnik, T., Miklavčič, D., 2014. Electroporation-Based Technologies for Medicine: Principles, Applications, and Challenges. Annu. Rev. Biomed. Eng. 16, 295-320. doi:doi:10.1146/annurev-bioeng-071813-104622

Yuxiang, Y., Minhang, K., Yong, L., Jian, W., Jing, Y., Zonghai, G., 2011. Design of a wideband excitation source for fast bioimpedance spectroscopy. Meas. Sci. Technol. 22, 13001.

Zhan, Y., Martin, V.A., Geahlen, R.L., Lu, C., 2010. One-step extraction of subcellular proteins from eukaryotic cells. Lab Chip 10, 2046-2048. doi:10.1039/c005152g 


\section{Supplementary material}

a) 450
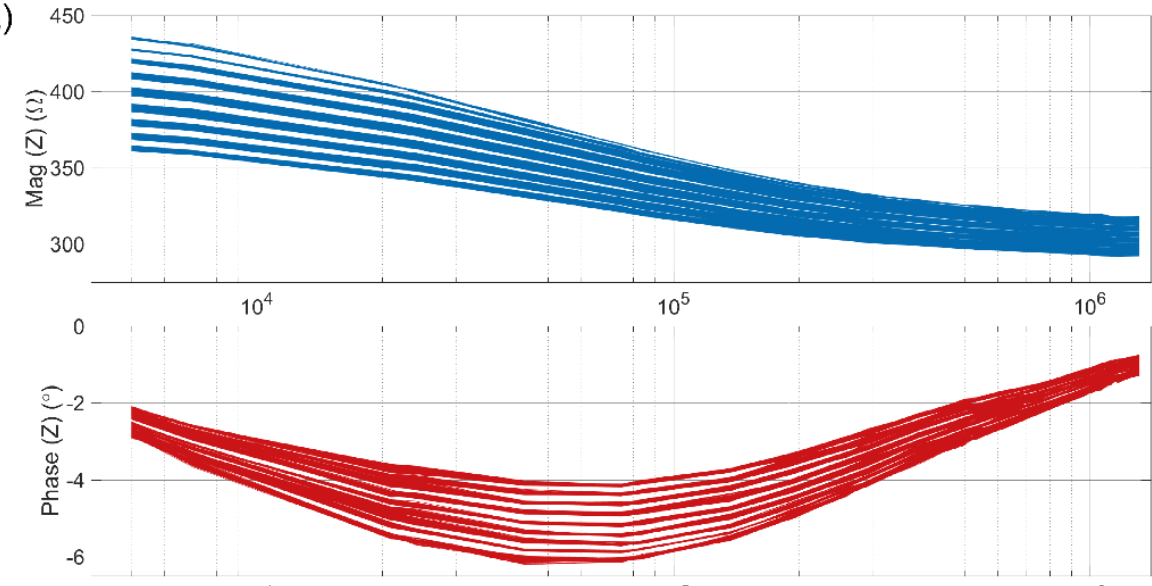

$10^{4}$

Frequency $(\mathrm{Hz})$

b)
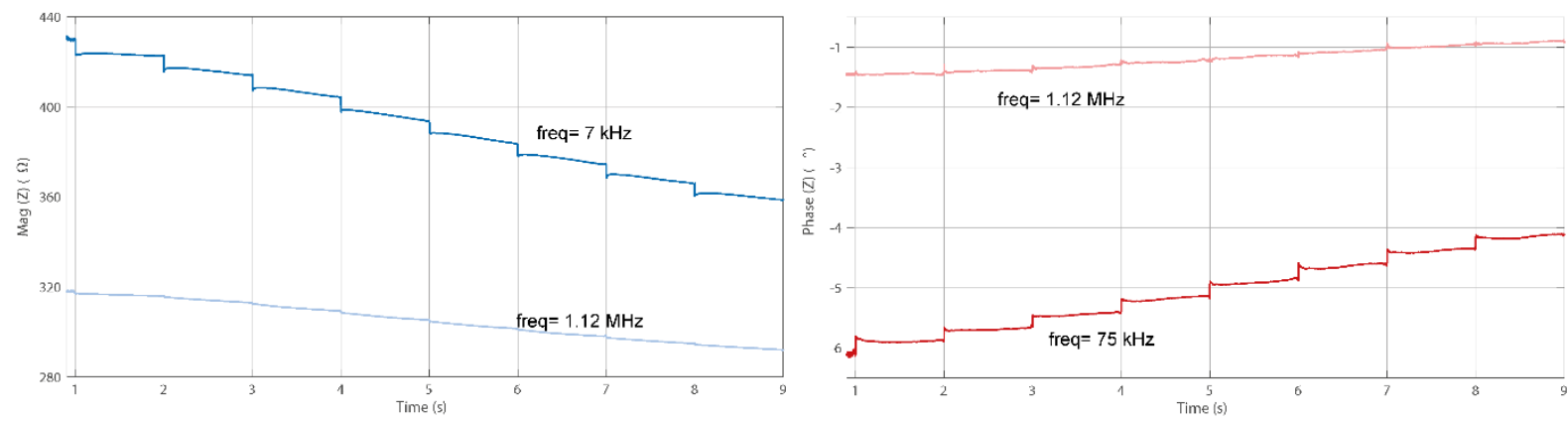

Fig. A Impedance spectrum evolution for a representative example of Hela cells exposed to an electric field intensity of $1000 \mathrm{~V} / \mathrm{cm}$. a) Bode plots showing the evolution in the impedance spectrum (magnitude and phase) pulse after pulse for a complete 8 pulses exposure experiment. b) Detailed temporal evolution in the impedance at two specific frequencies: $7 \mathrm{kHz}$ and $1.12 \mathrm{MHz}$ for the magnitude, and $75 \mathrm{kHz}$ and $1.12 \mathrm{MHz}$ for the phase 